VOX PATRUM 24 (2004) t. 46-47

Luciana Maria MIRRI

(Bologna)

\title{
GIROLAMO E I VESCOVI DI ROMA: UN RAPPORTO DI STIMA E DI COLLABORAZIONE
}

Se san Girolamo fosse nato nel 331, come alcuni studiosi hanno sostenuto, nell'arco della sua lunga esistenza sarebbe stato testimone del ministero petrino di ben dieci Pontefici: S. Silverio I romano (314-335), S. Marco romano (336), S. Giulio I romano (337-352), S. Liberio romano (352-366), S. Damaso spagnolo (366-384), S. Siricio romano (384-399), S. Anastasio romano (399401), S. Innocenzo I di Albano (401-417), S. Zosimo greco (417-418), S. Bonifacio I romano (418-422).

Collocando, invece, la sua nascita verso il 347 , tesi oggi la più avvallata, la sua vita avrebbe avuto inizio sotto il papato di Giulio I e la sua conversione sarebbe avvenuta con quello di Liberio, il vescovo di Roma che forse gli amministrò il battesimo verso il 366 .

Ma è durante il pontificato di papa Damaso che la vita e l'opera di Girolamo raggiunge il suo momento più felice: tra il 375 e il 378 vive l'esperienza ascetica nel deserto di Calcide, dove apprende la lingua ebraica e, con tutta probabilità, scrive la sua prima opera: la Vita Sancti Pauli Primi Eremitae, monachi Thebaei ${ }^{1}$, viene ordinato presbitero ad Antiochia dal vescovo Paolino fra il 377 e il 378; frequenta a Costantinopoli le lezioni di Gregorio di Nazianzo; entra in amicizia con Gregorio di Nissa e certamente partecipa al fermento del grande Concilio ecumenico di Costantinopoli I nel $381^{2}$. L'anno successivo è a Roma e diviene segretario particolare, archivista, consigliere e, soprattutto, amico personale del papa spagnolo il quale, intuendo il genio e valorizzando le capacità del monaco dalmata, gli commissiona la traduzione

${ }^{1}$ Girolamo stesso, concludendo il De viris illustribus, nel proprio medaglione cita per primo, tra le sue opere, questo scritto: cf. De viris illustribus 130, PL 23, 760. Per la Vita S. Pauli Primi Eremitae, cf. B. Degórski, Edizione critica della „Vita Sancti Pauli Primi Eremitae” di Girolamo, Roma 1987; idem, Przekaz lacińskich rękopisów Vita sancti Pauli Primi Eremitae św. Hieronima, Lublin 2000.

${ }^{2}$ Cf. L.M. Mirri, Girolamo, vivace protagonista del documento cristiano, in: Comunicazione e ricezione del documento cristiano in epoca tardoantica (Studia Ephemeridis Augustinianum 90), Roma 2004, 391-424. 
latina dei Vangeli ${ }^{3}$ e del Salterio e lo stimola ad altre interessanti esegesi. Nasce la versione Vulgata della Bibbia, che resterà per secoli quella ufficiale della Chiesa in Occidente a edificazione di generazioni di fedeli, e sorgono anche, sull'Aventino, i circoli culturali e monastici femminili di donne aristocratiche postisi sotto la guida spirituale e la scuola esegetica di quell'insigne asceta così stimato dal papa poeta 4 .

Dopo la morte di san Damaso, la vita di Girolamo ha una brusca svolta: privo del suo autorevole protettore, si impone l'esilio obbligatorio da Roma, dove troppo egli si è esposto inveendo contro la corruzione morale di clero e di nobili, in nome del Vangelo e della verità ${ }^{5}$. Ma i 35 anni di soggiorno a Betlemme che seguiranno saranno fortunatamente la continuità feconda di quanto seminato e coltivato durante il pontificato di Damaso: vita ascetica, monasteri femminili e maschili, e indefesso lavoro esegetico sulle Sacre Scritture ${ }^{6}$ o contro gli eretici fruttificheranno abbondantemente sull'esperienza romana precedente, benché purtroppo tra molte spine.

Il pontificato di Siricio romano vede Girolamo pellegrino in Terra Santa e in Egitto, seguito da alcune fedelissime discepole come Paola ed Eustochio, e poi residente a Betlemme ${ }^{7}$, tutto dedito a stilare diversi Commentari biblici, opere di questioni sui nomi ebraici, le Vite di Ilarione e Malco, altri due eremiti ${ }^{8}$, il De viris illustribus ${ }^{9}$, un'opera contro l'eretico Gioviniano e, soprattutto, a completare la Vulgata con i libri veterotestamentari.

Papa Anastasio governa brevemente la Chiesa, ma sono gli anni nei quali Girolamo è coinvolto nella crisi origenista che da tempo turba la cristianità specialmente in Oriente ${ }^{10}$. il momento dello scontro con Rufino e dei contatti con Teofilo, patriarca di Alessandria, che riceverà una lettera di papa Anastasio, inserita nell'epistolario geronimiano, in condanna finalmente dell'origenismo $^{11}$.

${ }^{3}$ Cf. G. Peters, I Padri della Chiesa, Roma 1986, 229-237, con tratti biografici di Girolamo. A pag. 238 è riportato qualche brano della lettera dedicatoria di Girolamo a papa Damaso, posta a Introduzione ai Vangeli: „Con del vecchio tu mi obblighi a fare del nuovo? Come arbitro, devo confrontare esemplari della Scrittura sparsi in tutto il mondo. Siccome divergono tra di loro, sono incaricato di decidere quali sono quelli che concordano con l'originale greco (dei Vangeli) [...]. Giudicare gli altri quando si è noi stessi sottoposti al giudizio di tutti! [...]. Il primo venuto [...] griderà che io sono un falsario e un sacrilego [...]. Perché il mio testo non sia troppo differente dalle letture latine a cui sono abituati, ho fermato la mia penna". Per la Vulgata cf.: Novum Testamentum D.N.J.C., latine secundum editionem Hieronymi, pars I, fasc. 1-5, Oxford 1889-1898

${ }^{4}$ Cf. L. Mirri, La dolcezza nella lotta. Donne e ascesi secondo Girolamo, Bose 1996, 149-165.

${ }^{5}$ Cf. Mirri, ibidem, p. 63-84.

6 Cf. Mirri, ibidem, p. 277-310.

7 Cf. Mirri, ibidem, p. 167-184.

${ }^{8}$ Cf. B. Degórski, Girolamo. Vite degli eremiti Paolo, llarione e Malco, Roma 1996.

${ }^{9}$ Per il testo italiano utilizzato nel presente lavoro cf. Girolamo, Gli uomini illustri, ed. E. Camisani, Roma 2000.

${ }^{10}$ Cf. S. Cola, Girolamo. Le lettere, vol. 3, Roma 1962, 7-22. 
Il pontificato di Innocenzo I coglie Girolamo ancora nel pieno impegno della lotta per l'ortodossia, oltre che in quello di carità per accogliere i profughi del sacco di Roma perpetrato da Alarico il 24 agosto del 410. Girolamo, che già ha perso a Betlemme santa Paola ${ }^{12}$, deve ora piangere la morte di altri amici rimasti nell'Urbe: Pammachio e Marcella. Inoltre, a causa delle sue arringhe contro i pelagiani, subisce da parte degli eretici la devastazione dei monasteri di Betlemme, dopo che anche il vescovo di Gerusalemme aveva assolto Pelagio. Nell'infausta circostanza, egli medesimo rischia la vita. Papa Innocenzo però manifesta solidarietà a Girolamo e riprende severamente Giovanni di Gerusalemme per il suo operato ${ }^{13}$.

San Zosimo regna come vescovo di Roma circa un anno: Girolamo è vecchio e stanco. Il pontificato brevissimo di questo pontefice greco gli consente almeno di bilanciare tante sofferenze, cui si aggiunge pure il dolore per la morte della diletta Eustochio, con la gioia di vedere legittimamente salire sulla Cattedra di Pietro un suo amico, Bonifacio, al quale commosso può esprimere, tra le lacrime del recente lutto, la propria consolazione per quell'ultima notizia lieta che la sua vita raccoglie, prima di spegnersi il 30 settembre del 420 , presso la grotta della Natività del Signore. Qui, con la sua nascita al cielo, Girolamo entra dunque in piena comunione dei santi anche con quei vescovi di Roma vissuti prima del suo tempo e la considerazione per i quali gli aveva fatto compilare, seppur in breve, uno schizzo di memoria tra gli uomini illustri. Essi sono: S. Pietro medesimo, S. Clemente romano, S. Vittore I, e S. Cornelio I, che entrano pertanto a far parte dei ,papi di Girolamo", almeno per quanto egli, di loro come vescovi di Roma, ha lasciato tracciato di significante ricordo.

${ }^{11}$ Cf. Hieronymus: $E p .82$, CSEL 55, 107-119 (a Teofilo in autodifesa contro Rufino); $E p .86$, CSEL 55, 138-139 (a Teofilo di felicitazioni per la sua posizione contro l'origenismo); Ep. 88, CSEL 55, 141-142 (a Teofilo sull'Occidente e l'origenismo). Cf. ancora, nell'epistolario geronimiano: Ep. 87, CSEL 55, 140 (a Girolamo, di Teofilo, sull'origenismo nei monasteri di Nitria); Ep. 89, CSEL 55, 142-143 (a Girolamo, di Teofilo, con invito a smascherare gli origenisti anche in Palestina); Ep..95, CSEL 55, 157-158 (a Simpliciano, di papa Anastasio con approvazione dell'operato di Teofilo che ha condannato l'origenismo). Nell'epistolario figurano altre lettere dello stesso periodo e sulla medesima controversia. Tra queste segnaliamo anche l'Ep. 81, CSEL 55, 106-107 (di Girolamo a Rufino contro la sua prefazione al Peri Archôn di Origene).

12 Cf. Mirri, La dolcezza nella lotta, p. 139-148: la discepola santa.

13 Cf. Ep. 85, CSEL 56, 263 (di papa Innocenzo ad Aurelio sui tragici fatti di Betlemme); Ep. 136, CSEL 56, 263-264 (di papa Innocenzo a Girolamo) ed Ep. 137, CSEL 56, 264-265 (di papa Innocenzo a Giovanni di Gerusalemme). Girolamo scriverà anche a sant'Agostino con elogi per la sua vittoria contro i pelagiani: cf. Ep. 141, CSEL 56, 290-291 ed Ep. 142, CSEL 56, 291-292. Infine, il Dottore dalmata ha più opere polemiche sulla questione: Contra Joannem Hierosolymitanum, PL 23, 371-412; Apologia contra Rufinum libri duo, PL 23, 415-478; CCL 79, 1-72; SCh 306, 6-199; Epistula adversus Rufinum, PL 23, 477-514; CCL 79, 73-116; SCh 303, 212-335; Adversus Pelagianos libri tres, PL 23, 517-618. 


\section{VESCOVI DI ROMA „VIRI ILLUSTRES”}

È certamente interessante prendere in considerazione i medaglioni biografici di Girolamo sui vescovi di Roma da lui inseriti tra gli Uomini illustri, perché se ne possono trarre eloquenti elementi sulla visione che egli ha di colui che occupa la Cattedra di Pietro e sul ministero petrino in sé. Escludendo per ora dal gruppo san Damaso, suo contemporaneo, quattro restano le figure alle quali volgere l'attenzione nell'opera citata del Dalmata.

1. Simone Pietro. Scorrendo l'epistolario geronimiano, mentre sono moltissimi i riferimenti a Pietro l'Apostolo ${ }^{14}$, soltanto due sono invece le lettere che lo menzionano - e una volta sola ciascuna - con il nome per esteso di Simone Pietro, quello cioè che il santo Dottore utilizza per presentarlo in apertura dei suoi Uomini illustri.

Il contesto si rivela subito non gratuito al dato: nell'Epistola $(=E p)$. a Edibia, Girolamo risponde al quinto quesito di dodici domande esegetiche postigli e deve trattare della frase di Gesù in $G v 20,17$, rivolta a Maria Maddalena: „Non toccarmi, perché non sono ancora salito al Padre mio”; nell' $E p$. 122 a Rustico, sul tema della penitenza, egli pone una bella introduzione sulla vocazione dei primi Apostoli e sul loro primo incontro con il Nazareno. Dunque, si tratta di due contenuti cruciali del Vangelo: la testimonianza del Risorto e la sua sequela, punti fondanti l'opzione di fede del cristiano.

Nell'Ep. 120, accingendosi alla sua esposizione, Girolamo riassume l'antefatto ricordando:

„Come s'accorse [Maria Maddalena] che la pietra che chiudeva il sepolcro era stata rimossa, «andò di corsa da Simon Pietro» e da quell'altro discepolo che Gesù amava in modo particolare"15.

Evidente è l'impiego del racconto di Gv 20, 2 parafrasato con il medesimo linguaggio tecnico dell'evangelista: il primo tra gli Apostoli viene appellato con il suo nome d'origine e con quello della nuova chiamata in ,sequela Christi”, consistente nel dover essere il riferimento di convalida della fede dei condiscepoli. L'ora della Risurrezione è l'ora di effettiva entrata in vigore del primato di Pietro e del suo ministero secondo il mandato di Gesù: „Conferma nella fede i tuoi fratelli" ( $\operatorname{Lc} 22,32)$. Parlando di quel momento, allora, origine del kerigma cristiano con l'annuncio pasquale, anche Girolamo non abbrevia i dati principali e i ruoli dei protagonisti: il pescatore di Cafarnao da quell'istante sarà per sempre la Roccia dei discepoli di Cristo. Il ministero petrino è,

\footnotetext{
${ }^{14}$ Cf. Index 3, Petrus (ap.), CSEL 56/2, 256-257.

15 Ep. 120 5, CSEL 55, 484: „cumque lapidem, quo monumentum clausum fueret, vidisset ablatum, cucurrit ad Simonem Petrum et ad alterum discipulum, quem Iesus amabat plurimum".
} 
dunque, per l'eremita di Betlemme, innanzi tutto annuncio, conferma e garanzia del nucleo caratterizzante e cardine fondante del credo cristiano: la Risurrezione del Cristo.

Nell'Ep. 122, l'esordio è un encomio allo zelo apostolico di Edibia ${ }^{16}$ e di Artemia $^{17}$. Il destinatario della lettera non risulta conosciuto personalmente da Girolamo, il quale gli dice francamente:

„Non mi conosci e non ti conosco, ma se mi permetto di scriverti è grazie alle insistenti preghiere di Edibia [...] nonché di Artemia [...] smaniosa di imitare lo zelo affettuoso degli Apostoli Andrea e Filippo i quali, scovati ambedue da Cristo, non hanno che un desiderio: andare a pescare anch'essi il fratello Simone l'uno, e l'amico Natanaele l'altro. Simone, così, meriterà di sentirsi dire: «Tu sei Simone, figlio di Giovanni? Ti chiamerai Cefa, che significa Pietro" (Gv 1, 42)"18.

Le parole del santo Dottore sono significative nell'uso misurato dei termini coi quali descrive l'assunto evangelico. Andrea e Filippo, „scovati” dal Signore, sono i primi ,pescatori di uomini” e, in tal caso, si può dire di altri Apostoli, trattandosi di Pietro e di Natanaele. Grazie a loro, anche Simone sarà „pescato" da Cristo e riceverà la grande chiamata. L'unico „merito” del pescatore di Cafarnao sembra essere quello di essersi lasciato pescare da Gesù, tramite l'entusiasmo missionario degli altri due discepoli.

Nella scheda dedicatagli da Girolamo nel De viris illustribus, i suoi dati anagrafici e cristiani sono lapidari: „Simone Pietro, figlio di Giovanni (= Giona), nato a Betsaida, in Galilea, era fratello dell'apostolo Andrea (cf. $M t 4,18$ ) e capo degli Apostoli" 19 . Le altre informazioni fornite dal monaco dalmata appartengono alla tradizione del suo tempo prima che al riscontro storico di prove certe tuttora mancanti. Pietro è considerato „vescovo di Antiochia” e poi, portatosi ,a Roma per debellare Simone mago, ivi occupò la cattedra episcopale per venticinque anni, fino all'ultimo anno di Nerone, vale a dire fino al quattordicesimo anno del suo regno" ${ }^{20}$.

${ }^{16}$ Cf. Mirri, La dolcezza nella lotta, p. 120-122: era una nobile vedova della Gallia corrispondente di Girolamo.

${ }^{17}$ Cf. Mirri, ibidem, p. 99-101: sposa di Rustico, andata dalla Gallia a Betlemme.

18 Ep. 122, 1, CSEL 56, 56: „Quod ignotus ad ignotum audeo scribere, sanctae ancillae Christi Hedybiae [...] et Artemiae [...] fecit deprecatio. Quae [...] imitari cupiens Andreae et Philippi Apostolorum benivolentiam, quorum uterque inventus a Christo fratrem Simonem et amicum Nathanahel invenire desiderat, ut alter eorum mereatur audire: tu es Simon, filius Iohannis, tu vocaberis Cephas, quod interpretatur Petrus".

${ }^{19}$ De viris illustribus I, PL 23, 638: „Simon Petrus, filius Joannis, provinciae Galileae, e vico Bethsaida, frater Andreae apostoli, et princeps apostolorum".

${ }^{20}$ Ibidem: „post episcopatum Antiochensis Ecclesiae [...] ad expugnandum Simonem magum, Romam pergit, ibiqui viginti quinque annis cathedram sacerdotalem tenuit, usque ad ultimum annum Neronis, id est, decimum quartum". Per Simon Mago cf. At. 8, 9-25. In PL 23, 637 note 
Dunque, Pietro risulterebbe vescovo di Roma dal 42 al 67, allorché ricevette la corona del martirio mediante crocifissione a capo in giù per il noto motivo di reputarsi indegno della medesima morte del Signore ${ }^{21}$, notizia che ha la sua fonte in Origene ed Eusebio. La storia dell'arte l'ha quindi codificata sia in Oriente che in Occidente, come icone e quadri famosi mostrano. Quanto al motivo di confutazione dell'operato ingannevole di Simon mago, causa del trasferimento di Pietro da Antiochia a Roma, le perplessità sono legittime. Più valido è il pensiero che sotto questa presentazione di causa importante si celi la messa in gioco di confutazione di idolatria, magia pagana oppure di difesa del carisma dei discepoli e del ministero apostolico nella nascente gerarchia e comunità cristiane. L'opera di Pietro è comunque intesa sotto una luce apologetica della verità e di rettifica di abusi o errori, non solo in materia di fede, bensì pure organizzativa nella vita della Chiesa primitiva. Ciò sembra che, nell'idea geronimiana, costituirà il proprium del ministero petrino, in base a quanto evidenzierà di altri vescovi di Roma.

Vengono infine menzionati, di Pietro, gli scritti ritenuti autentici e quelli certamente spuri, come la seconda lettera appartenente ai testi ispirati, oppure apocrifi evidentemente in circolazione, tanto che Girolamo ne cita uno sconosciuto: il Giudizio 22 . Data l'importanza dell'Apostolo Pietro, non è consentito barare sulla veridicità di opere passate sotto suo nome: se il Dalmata è intransigente riguardo alla letteratura apocrifica in $s^{23}$, ancor più si dimostra intollerante nell'attribuzione al capo degli apostoli di pagine inconsistenti per la fede e, forse, per questo si fa scrupoloso ed esauriente nel suo elenco di falsi attribuiti a Simon Pietro: l'inganno entrerebbe nella genuina tradizione della comunità dei credenti, non senza grave danno.

Quanto al primo vescovo dell'Urbe, egli conclude la presentazione dichiarando che Pietro „fu sepolto a Roma, sul colle Vaticano" e che „è oggetto di culto universale" 24 , parole che rimandano palesemente al ruolo cattolico avuto in vita dal primo tra gli Apostoli.

2. Clemente Romano. A seguito di medaglioni biografici di Apostoli ed Evangelisti, Girolamo inserisce qualche autore latino e quindi inizia la sequen-

riportano altri riferimenti di Girolamo e di Eusebio sulla notizia per la quale „primum episcopum Antiochenae Ecclesiae Petrum fuisse accepimus" (cf. Girolamo, In Gal. I 2, PL 26, 366).

${ }^{21}$ Cf. De viris illustribus 1, PL 23, 638: „martyrio coronatus est, capite ad terram verso, et in sublime pedibus elevatis; asserens se indignus qui sic crucifigeretur ut Dominus suus".

22 Cf. De viris illustribus 1, PL 23, 639: „Libri autem, e quibus unus Actorum ejus inscribitur, alius Evangelii, tertius Praedicationis, quartus Apocalypseos, quintus Judicii, inter apocryphas scripturas repudiantur".

${ }^{23}$ Cf. Hieronymus, Adversus Helvidium 8bis, PL 23, 201: „Quae sententia et apocryphorum deliramenta convincit".

${ }^{24}$ Cf. De viris illustribus 1, PL 23, 639: „Sepultus in Romae in Vaticano, [...] totius orbis veneratione celebratur". 
za di alcuni Padri Apostolici: di questi ultimi, Clemente è il secondo, tra il Pastore di Erma ed Ignazio di Antiochia. Di lui dice:

„Fu il quarto vescovo di Roma dopo Pietro; infatti, il secondo fu Lino e il terzo Anacleto, anche se molti latini ritengono che Clemente fu il secondo dopo Pietro"25.

Il monaco dalmata non lesina le informazioni ricevute dalla tradizione e, in assenza di dati certi, ne riporta al completo i contenuti. Il fatto di ritenere Clemente il secondo vescovo di Roma risale con tutta probabilità all'opinione che fosse stato consacrato da Pietro stesso, secondo una notizia di Tertulliano. Epifanio tuttavia fu colui che precisò come per amore della pace Clemente avesse rinunciato alla cattedra petrina in favore prima di Lino e poi di Anacleto, onde evitare tensioni nella comunità. In sostanza, si sottolineano in ogni modo il nesso diretto con Pietro e, dunque, la più legittima ed autentica successione apostolica, e la capacità santa di Clemente di agire con umiltà in favore dell'unità dei credenti attorno al pastore.

Meno probabile è invece il dato che Clemente papa sia il medesimo Clemente citato da san Paolo in Fil 4, 3. Origene ed Eusebio tramandano ciò, ma con difetto di prove. Palese è pertanto la dipendenza di Girolamo da quesi due autori, con la preoccupazione di fornire dettagli in più su di un personaggio importante, ma sul quale tuttora poco si conosce di sicuro. Il santo Dottore sembra intento tra le righe a codificare l'assunto di una successione autentica da Pietro a Clemente, anche tramite gli anelli di Lino e di Anacleto per altro entrati in tale sequenza nel canone romano della liturgia eucaristica latina.

Girolamo pone in risalto il dettame altamente apostolico di un vescovo di Roma connesso sia al primo degli Apostoli, Pietro medesimo, sia all'Apostolo per antonomasia a motivo del suo ministero, Paolo. Il riferimento a Fil 4, 3, dove quest'ultimo accenna a collaboratori così valenti nell'opera di evangelizzazione da avere i nomi „scritti nel libro della vita", è un elogio di immediata presentazione della santità e dello spessore del personaggio in questione, sia che si tratti di Clemente papa, sia che si riferisca Paolo ad altro omonimo ministro. Per Girolamo quelle parole sono applicate al quarto vescovo di Roma e tanto basta per presentarlo più che autorevolmente. Quindi, di lui, afferma: „A nome della Chiesa romana, egli scrisse una Lettera alla Chiesa di Corinto, lettera quanto mai preziosa, che in varie parti si legge anche pubblicamente" 26 . La formula adottata dal nostro Autore è interessante: Clemente scrive alla Chiesa di Corinto a nome della Chiesa di

${ }^{25}$ De viris illustribus 15, PL 23, 663: „Clemens [...] quartus post Petrum Romae episcopus: siquidem secundus Linus fuit, tertius Anacletus, tametsi plerique Latinorum secundum post Petrum apostolum putent fuisse Clementem".

${ }^{26}$ De viris illustribus 15, PL 23, 663: „Scripsit ex persona Romanae Ecclesiae ad Ecclesiam Corinthiorum valde utilem Epistolam, quae et in nonnullis locis publice legitur". 
Roma, e non suo proprio. Viene così sottolineata la collegialità del ministero petrino nell'esercizio comunque di un primato almeno morale nell'invocare l'unità ecclesiale e di fede delle comunità. Questo servizio in specifico all'episcopè in seno alla catholica è la testimonianza che impreziosisce il documento, scritto forse tra il 95 e il 98, e che, secondo Dionigi vescovo di Corinto, era effettivamente letto durante la liturgia della domenica, avvallando l'informazione di Girolamo.

Dalle poche righe del Dalmata risulta pertanto che Clemente, legittimo successore di Pietro, possedeva ed esercitava le qualità apostoliche per vegliare sull'intero gregge del Signore. Il suo ministero è sollecito non solo per l'Urbe, ma anche fino in Oriente, là dove una comunità vive una crisi ed è disorientata, con grave pericolo di fede, unità e carità. La sua abilità di uomo di pace insieme ad una non comune virtù di profonda umiltà e di forte spirito di servizio, fa sì che in Clemente il carisma di Pietro maturi nello sviluppo d'un primato che esercita la funzione di guida del popolo di Dio unito in pascoli tranquilli e di un ministero caratterizzato sempre più dal configurarsi in un essere servus servorum Dei, come Gregorio Magno con bella espressione definirà in seguito il suo compito di vescovo di Roma ${ }^{27}$.

Se quindi Girolamo non accetta l'attribuzione a Clemente romano della seconda lettera ai Corinti e di un altro scritto intitolato Disputa di Pietro con Apione, antica omelia cristiana composta probabilmente a Corinto verso il 140, non altrettanto si può dire riguardo alla Lettera agli Ebrei. Il suo ipotizzare favorevolmente la paternità di Clemente, già considerato discepolo di san Paolo, è esplicita, „,non solo riguardo al contenuto, ma anche per la forma stessa del periodare”, e ne evidenzia pertanto la „grandissima affinità" con quella clementina prima Lettera ai Corinti ${ }^{28}$.

Dunque, Clemente romano addirittura autore ispirato tra quelli neotestamentari? Tesi affascinante, che Girolamo accarezza con la sua autorità di biblista, forse atta questa volta a fornire i massimi elementi disponibili per àncorare un grande successore di Pietro, da lui molto apprezzato, non solo agli Apostoli martirizzati nell'Urbe sotto Nerone, ma anche alla Parola divina, di cui fu eccellente testimone con la santità di vita e il servizio ecclesiale,

${ }^{27}$ Cf. Gregorius Magnus, Ep. 6, 50. Gregorio comunque si definiva tale anche da semplice monaco.

${ }^{28}$ Cf. De viris illustribus 15, PL 23, 663-666: „quae mihi videtur characteri Epistolae, quae sub Pauli nomine ad Hebraeos fertur, convenire. Sed et multis de eadem Epistola, non solum sensibus, sed juxta verborum quoque ordinem abutitur. Omnino grandis in utraque similitudo est. Fertur et secunda ejus nomine Epistola, quae a veteribus reprobatur. Et Disputatio Petri et Appionis longo sermone conscripta". Per Clemente Romano, cf. anche: I Padri Apostolici, ed. A. Quacquareli, Roma 1978, 41-48; G. Peters, I Padri della Chiesa, Roma 1984, 56-84. Cf. anche: Giovanni Paolo II, Lettera Enciclica „Ut unum sint" (25 maggio 1995), nn. 88-97 (sul ministero d'unità del vescovo di Roma e la comunione di tutte le Chiese particolari con la Chiesa di Roma). 
prestato da Clemente fino all'anno terzo di Traiano, cioè il 101, allorché morì. Già al IV secolo, ,il ricordo del suo nome è conservato da una chiesa edificata in Roma"29.

Singolare è il silenzio di Girolamo sul presunto martirio di Clemente nel Chersonneso. Forse non ne ha notizia, non parlandone neppure Eusebio nella sua Storia Ecclesiastica e, comunque, a differenza di Pietro di cui afferma che „è sepolto sul colle Vaticano", per Clemente parla soltanto di memoria del suo nome, tenuta viva al sec. IV da una chiesa dedicatagli nell'Urbe, senza accennare che in essa vi siano venerate le sue spoglie. Ciò potrebbe essere già un indizio sulla non presenza delle reliquie di Clemente in Roma e sulla non conoscenza, all'epoca, del luogo in cui si trovassero, e si confermerebbe il loro ritorno nella Capitale nel sec. IX ad opera dell'Apostolo degli Slavi san Cirillo, che solo allora le avrebbe depositate nella citata basilica già dedicata a quel santo vescovo di Roma ${ }^{30}$.

3. Vittore. Nel capitolo 53 degli Uomini illustri, si legge: „Tertulliano, presbitero, appare qui finalmente come il primo degli scrittori latini, dopo Vittore ed Apollonio" "31. Pertanto, in realtà, primo degli scrittori latini, risulterebbe questo „tredicesimo vescovo di Roma”, che „scrisse Sulla questione della Pasqua" e che „compose alcuni altri opuscoli, e resse la Chiesa per dieci anni sotto l'imperatore Severo (193-211)"32.

Dunque, al capitolo 34 che gli dedica, Girolamo sembra volere in effetti sottolineare quasi esclusivamente l'opera letteraria di questo papa, per il quale non spende altre parole. Gli attribuisce, in particolare, anche delle lettere scritte sempre in relazione alla controversia circa la data di Pasqua, e delle quali riferisce pure Eusebio. Probabilmente, ciò si deve al fatto che verso la fine del sec. II, proprio con Vittore viene alla ribalta l'elemento latino: fin lì, ogni scritto cristiano, è quasi esclusivamente in lingua greca. Perciò, a questo vescovo di Roma che fu tale circa dal 189 al 198, va il merito non di aver stilato opere letterarie, ma di aver introdotto in qualche modo, con la sua autorità di successore di Pietro, la lingua latina nelle questioni ecclesiastiche. Girolamo non è un teologo, bensì un biblista: forse è questo il motivo che l'induce a tacere la scomunica inflitta da Vittore a Teodoto di Bisanzio in ordine alla eresia

29 De viris illustribus 15, PL 23, 666: „Obiit tertio Trajani anno, et nominis ejusmemoriam usque hodie Romae exstructa Ecclesia custodit". Altri riferimenti a Clemente Romano, nell'epistolario di Girolamo, sono: Ep. 49, 19, CSEL 54, 383 ed Ep. 129, 3, CSEL 56, 169.

30 Anche san Cirillo venne poi jvi sepolto.

31 De viris illustribus 53, PL 23, 698: „Tertullianus presbyter, nunc demum primus post Victorem et Apollonium Latinorum ponitur".

${ }^{32}$ De viris illustribus 34, PL 23, 682: „Victor, tertius decimus Romanae urbis episcopus, super quaestione Paschae et alia quaedam scribens opuscula, rexit Ecclesiam sub Severo principe anni decem". 
adozionista e a trattare succintamente solo dei suoi interventi nella disputa della data di Pasqua ${ }^{33}$.

Secondo Eusebio ${ }^{34}$, papa Vittore aveva sollecitato sinodi episcopali in Ponto, Palestina e Siria per risolvere definitivamente la diatriba. Il problema, tuttavia, rimase purtroppo dolorosamente aperto fino al Concilio di Nicea I (325), con non poche tensioni tra le diverse regioni cristiane. I dati brevissi$\mathrm{mi}$, quasi laconici, fornitici pertanto da Girolamo pare vogliano porre l'attenzione unicamente sul ruolo che un vescovo di Roma ha avuto in rapporto alla tradizione latina nella Chiesa, introducendo l'uso del latino in documenti propri ufficiali, e quindi nel primo insorgere di controversie minaccianti fede, culto delle comunità ecclesiali, in specifico il cuore dell'una e dell'altro: la celebrazione della Pasqua del Signore. Anche soltanto per questo intervento, Vittore I quale successore di Pietro ha meritato, nella considerazione del monaco di Betlemme, un posto tra gli uomini illustri, seppur di tre righe appena per tramandarne memoria rilevante.

4. Cornelio. Il suo nome è legato a quello di Cipriano, che morì „nello stesso giorno, ma non già nello stesso anno, di Cornelio a Roma"35. Il giorno è il 14 settembre, l'anno del martirio di Cipriano è il 258 , mentre quello della morte in esilio a Civitavecchia di Cornelio è il 253. Girolamo, però, come tutta la tradizione, attribuisce pure a questo vescovo di Roma, al quale „successe Lucio [...] il martirio per la fede in Cristo" ${ }^{36}$. E come quello del suo successore, anche il suo pontificato fu assai breve: „Amministrò la Chiesa per due anni sotto Gallo e Volusiano"37, vale a dire dal 251 al 253.

L'elenco, comunque, dei suoi scritti narra da sé quanto sia stato intenso e drammatico il ministero petrino di questo vescovo di Roma, ed è appunto con tale informazione sintetica che Girolamo ce ne può lasciare un ottimo ritratto biografico. Dopo aver accennato alle „otto lettere di Cipriano tuttora esistenti" a lui indirizzate, ed incluse nell'epistolario del noto vescovo di Cartagine, il santo Dalmata aggiunge che „Cornelio, vescovo di Roma [...] scrisse una lettera A Fabio, vescovo di Antiochia, intorno ai sinodi di Roma, d'Italia e dell'Africa, un'altra Su Novaziano; una terza Sui lavori del Sinodo, una quarta Al medesimo Fabio, quanto mai estesa e riguardante le cause e le condanne della setta Novaziana"38. La storia ci informa che l'indefesso ministero di Cornelio dovette fronteggiare innanzi tutto un serio pericolo di scisma al centro della

${ }^{33}$ Cf. K. Baus, Storia della Chiesa. Le origini, vol. I, ed. H. Jedin, Milano 1988, 350-357, $452-457$.

34 Cf. Eusebius, HE V 23 e V 16.

${ }^{35}$ De viris illustribus 67, PL 23, 714: ,[Cyprianus Afer] Passus est sub Valeriano et Galieno principibus, persecutione octava, eodem die quo Romae Cornelius, sed non eodem anno".

${ }^{36}$ De viris illustribus 66, PL 23, 714: „[...] cui ob Christum martyrio coronato successit Lucius”.

37 Ibidem: „Rexit Ecclesiam annis duobus sub Gallio et Volusiano”. 
Cristianità: l'elezione illegittima di un antipapa a Roma nella persona di Novaziano che, per altro, si presentava anche come il primo teologo utilizzante il latino e non il greco. Quindi, nei mesi successivi la sua elezione, dovette impegnarsi contro lo stesso per arginare idee intransigenti sul perdono dei lapsi.

Girolamo, nel cap. 70 del De viris illustribus, di Novaziano racconta che fu „prete della Chiesa romana”, il quale „mirò ad occupare la cattedra episcopale in opposizione a Cornelio, introdusse l'eresia detta - con termine greco 'dei Catari'. Egli non voleva riammettere nella Chiesa gli apostati, anche dopo la loro penitenza"39. Dunque, apponeva un rigorismo estremo alla prassi penitenziale della Grande Chiesa, orientata invece al primato della carità e della misericordia. Causa seguaci di Novaziano, molti morirono in disperazione per la loro salvezza e non solo lapsi, bensì pure alcuni che erano incorsi in peccato mortale. Per debellare subito la diffusione dell'eresia novaziana, papa Cornelio indisse sinodi locali affinché nelle province in cui andavano propagandandosi tali idee errate fosse quanto prima ristabilita l'ortodossia della fede. È il contenuto della prima lettera a Fabio e della terza, quella dove si accenna anche al sinodo che si svolse a Roma nel 251, in cui 60 vescovi presieduti da Cornelio pronunciarono la scomunica di Novaziano e dei suoi seguaci ${ }^{40}$.

D'altronde, la scissione con l'impostore che mirava ad usurpare la cattedra di Pietro era ormai diventata insanabile: al medesimo confratello nell'episcopato residente in Antiochia, Cornelio scriverà perciò pure la quarta lettera per chiarire definitivamente l'accaduto e i motivi delle gravissime decisioni prese.

Ed è in uno di questi scritti del 251 a Fabio che Cornelio illustra l'organizzazione e la consistenza dei ministeri nella Chiesa romana, che poteva avvalersi di 154 membri del clero, suddivisi in: 46 presbiteri, 7 diaconi, 7 suddiaconi, 42 accoliti e 52 tra esorcisti, lettori ed ostiari. Inoltre, 1500 erano le vedove e i poveri assistiti dalla solidarietà della comunità ecclesiale dell'Urbe ${ }^{41}$.

Si può pertanto concludere che in due anni di pontificato, il ministero petrino di Cornelio sia stato attivo efficacemente sia nella lotta acerrima finalizzata a preservare l'unità gerarchica e di fede della Chiesa, sia a promuovere la carità evangelica in seno alla stessa, in particolare a Roma, quasi perché

38 Ibidem: „Cornelius, Romanae urbis episcopus, ad quem octo Cypriani exstant epistolae, scripsit epistolam ad Fabium Antiochenae Ecclesiae episcopum, de synodo Romana, Italica, Africana, et aliam de Novatiano, et de his qui lapsi sunt; tertiam de gestis synodi; quartam ad eumdem Fabium valde prolixam, et Novatianae haereseos causas et anathema continentem".

39 De viris illustribus 70, PL 23, 715: „Novatianus, Romanae urbis presbyter, adversus Cor-

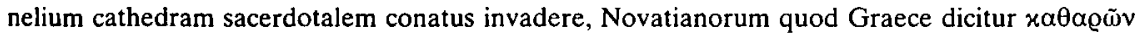
dogma constituit, nolens apostatas suscipere paenitentes".

${ }^{40}$ Cf. Baus, Storia della Chiesa, vol. I, p. 320-321.

${ }^{41}$ Cf. Eusebius, HE VI 43, 11; Baus, Storia della Chiesa, vol. I, p. 480-481. 
potesse questa offrirsi a modello e testimonianza delle comunità sorelle. Il martirio, dunque, di tale vescovo di Roma, sigla e corona, nell'ideale anche geronimiano, l'incisività santa dell'opera di un pastore che come Pietro è stato Roccia consolidante la fede dei fratelli in tempi di persecuzione non più solo esterna, ma purtroppo anche già interna alla Chiesa medesima.

\section{PAPI „BEATISSIMI AMICI”}

Tra il monaco dalmata, ,vir trilinguis”, e il papa spagnolo Damaso, poeta, come ancora tra il primo ormai esule volontario a Betlemme e Bonifacio, romano d'origine, la stima e la collaborazione scaturirono da assai più profonde radici di santa amicizia spirituale e umana. I toni di fine devozione reciproca, premura, sincera solidarietà nei successi come nelle sofferenze, e ogni tipo di sollecitudine e compartecipazione che emerge dalla corrispondenza di Girolamo, costituiscono una lettura immediata di una felicissima comunione tra uomini di Dio votati a servire fede e verità con tutto se stessi.

1. Damaso. Successore di Pietro dal 366 al 384, tra i suoi "meriti" non elencati dal santo Dottore c'è quello di aver intuito il genio riposto in quella forte personalità del monaco venuto a Roma nel 382 dall'Oriente e di averlo assunto a segretario personale. Pochi anni dopo la scomparsa dell'amico papa, dal suo ritiro di Betlemme, Girolamo così lo ricorderà tra gli Uomini illustri:

„Damaso, vescovo di Roma, ebbe un fine talento nell'arte poetica, e pubblicò numerose e brevi composizioni metriche. Morì, quasi ottantenne, sotto l'imperatore Teodosio" 42 .

Sintetico forse per non cedere a sentimenti ancora vivissimi di un intenso rapporto di stima e di collaborazione, Girolamo tuttavia in quei termini „elegans", „genium habuit" e „multa et brevia metro edidit" tradisce lo spessore di una considerazione non comune, specialmente se per l'ultima citazione si avvalla una tradizione di codici che anche recita: „multaque brevia opuscola heroico metro edidit" 43 , in consonanza con quanto indicato da Girolamo medesimo alla discepola Eustochio ${ }^{44}$. Purtroppo, di tali scritti di Damaso sulla verginità cristiana, nulla è pervenuto se non questa testimonianza del Dalmata,

${ }^{42}$ De viris illustribus 103, PL 23, 742: „Damasus, Romanae urbis episcopus, elegans in versibus componendis ingenium habuit, multaque et brevia metro edidit, et prope octogenarius sub Theodosio principe mortuus est".

43 Cf. PL 23, 741, nota 2.

44 Alla ragazza, egli suggerisce di leggere, tra l'altro, ,gli scritti di papa Damaso in versi e in prosa su questo argomento" della verginità cristiana, le angustiae nuptiarum e la libertà da esse della donna consacrata (cf. Ep. 22, 22, CSEL 54, 175). 
indice di una pastorale e di una spiritualità condivise dai due amici. Restano, invece, una sessantina di epigrammi in esametri composti dal papa spagnolo in onore dei martiri, altro elemento di comune interesse con Girolamo ${ }^{45}$.

Comunque, nell'epistolario geronimiano è conservato un certo carteggio intercorso tra le due interessanti personalità. Già dal deserto di Calcide Girolamo, non riuscendo a raccappezzarsi nei cavilli terminologici che a motivo di eresie trinitarie gli altri asceti continuamente gli sottopongono, tormentandolo sulla sua professione di fede in proposito, in „disperata semplicità" per quel tormento si rivolge al papa:

„[...] è difficile capire [...] per questo ho deciso di consultare la Cattedra di Pietro, dove si trova quella fede che la bocca di un Apostolo ha esaltato... solo presso di voi si conserva intatta la eredità dei padri" ${ }^{46}$.

La seconda parte della lettera è un'accorata supplica di Girolamo a Damaso, affinché si degni di prestare ascolto a lui che, „,come una pecorella” chiede „protezione al pastore”. Si trovano espressioni tali di riverenza da stupire nel temperamento sempre rude e sostenuto dell'asceta, il quale pure scrive:

„La tua grandezza, a dire il vero mi mette in soggezione, ma la tua bontà mi attira... sottraiti un momento al fasto dell'altissima dignità romana: è con il successore del pescatore e con un discepolo della croce che desidero parlare" ${ }^{47}$.

Con abile intuito, nell'ultima espressione, Girolamo definisce la funzione del ministero petrino nella veste più autentica di servo del Signore, e insieme il segreto per esercitarla all'insegna della santità: l'essere discepolo della Croce. La stima manifestata per papa Damaso si unisce quindi ad una professione di fede a Cristo e unità alla Chiesa come madre, con altrettanto efficaci parole:

"Io non seguo altro primato che quello di Cristo; per questo mi metto in comunione con la tua Beatitudine, cioè con la Cattedra di Pietro"48.

Altri vescovi, in Oriente, avrebbero potuto costituire per lui un riferimento, almeno per giurisdizione territoriale nella Chiesa di Antiochia, ma di loro Girolamo non si fida per diversi motivi e come neoconvertito vuole certezze assolute come netto è il suo principio: „Chi non raccoglie con te disperde; vale

${ }^{45}$ Cf. Mirri, La dolcezza nella lotta, p. 221-232.

46 Ep. 15, 1, CSEL 54, 62-63: „difficile, ubi fons signatus et hortus ille conclusus sit, possit intellegi, ideo mihi cathedram Petri et fidem apostolico ore laudatam censui consulendam [...] apud vos solos incorrupta patrum seruatur hereditas".

47 Ep. 15, 2, CSEL 54, 63: „Quamquam igitur tui me terreat magnitudo, tamen invitat humanitas [...] a pastore praesidium ovis flagito [...] Romani culminis recedat ambitio: cum successore piscatoris et discipulo crucis loquor".

48 Ibidem, CSEL 54, 63-64: „ego nullum primum nisi Christum sequens beatitudini tuae, id est cathedrae Petri, communio consocior". 
a dire: chi non è di Cristo è dell'anticristo"49. Girolamo, d'altronde sente la Chiesa di Roma come sua madre anche per avervi ricevuto il battesimo e il legame con il suo vescovo è dunque di per sé particolarissimo. Inoltre, in lui, si comincia a sentire l'attenzione al confronto con le Sacre Scritture, che gli sarà del tutto proprio e che non sarà sfuggito all'insigne corrispondente nell'Urbe. Dichiara infatti:

"[I cattolici di Antiochia] esigono da me, che sono romano, una nuova terminologia: 'le tre ipostasi'. Ma quale Apostolo, per favore, ha tirato fuori questa espressione?"50.

L'appello conclusivo dell'eremita di Calcide è quasi drammatico, dopo aver esposto senza timori e falsi pudori le proprie incomprensioni sulla questione dogmatica, ma ciò indica appunto una relazione filiale e confidente con chi rappresenta un padre nella fede:

"Scongiuro, dunque, la tua Beatitudine, per Gesù Crocifisso, salvezza del mondo, per la Trinità consustanziale, di autorizzarmi, mediante una lettera, a parlare di ipostasi oppure a non parlarne"

Pochi mesi dopo, non avendo ricevuto risposta, Girolamo replica aprendo la nuova lettera con vari riferimenti evangelici $(M t 15,21-28 ; L c 11,5-8 ; L c 19$, $8-9 ; L c 23,43)$. Gli accenti mutano in più intensa e sofferta passionalità: egli vuole salvare il suo battesimo restando nella verità, pur confessando di essere penitente giustamente per i peccati commessi e non disdegnando, infine, espressioni patetiche come nella conclusione: „Non trascurare un'anima per la quale è morto Cristo!"52. E continuando ad interpellare Damaso in seconda persona e con accento tra l'esortativo e l'imperativo di un dovere morale dice apertamente:

"Questo solo: desidero che tu, grande come sei, abbassi lo sguardo su questo povero uomo; che non disprezzi una pecorella malata, anche se come pastore ne hai altre a bizzeffe" 53 .

Mai più si vedrà Girolamo così implorante presso qualcuno; mai più alla sua penna usciranno espressioni nelle quali si stenta a riconoscere il Leone

49 Ibidem, CSEL 54, 64: „non novi Vitalem, Meletium respuo, ignoro Paulinum. Quicumque tecum non colligit, spargit, hoc est, qui Christi non est, antichristi est".

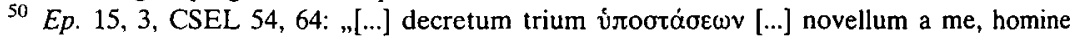
Romano, nomen exigitur. Qui ista, quaeso, apostoli prodidere?".

51 Ep. 15, 5, CSEL 54, 67: „Quam ob rem obtestor beatitudinem tuam per crucifixum, mundi salutem, per homousian trinitatem, ut mihi epistulis tuis sive tacendarum sive dictendarum hypostaseon detur auctoritas".

52 Ep. 16, 2, CSEL 54, 69: „noli despicere animam, pro qua Christus est mortuus".

53 Ep. 16, 1, CSEL 54, 68: "quorsum ista tam longo repetita prooemio? vidilicet, ut parvum magnus aspicias, ut dives pastor morbidam non contemnas ovem”. 
dalmata, sempre sicuro di sé, ironico contro eretici e nemici, fulminante con la sua conoscenza biblica e la tagliente parola. Damaso ha già avuto su di lui la più grande vittoria del bene dell'amicizia e della stima, della fiducia e della sapienza. Circa quattro anni dopo, una lunghissima lettera suddivisa in due parti è ancora indirizzata da Girolamo a Damaso. Forse sollecitata da quest'ultimo con molti quesiti, il Dalmata da Costantinopoli invia un testo che va considerato il più antico documento della sua capacità esegetica e contenente riflessioni tra le più belle in ordine alla spiritualità biblica delle sue opere.

Puntuale e persino elegante nel susseguirsi dell'esposizione, l'asceta debutta in quello che pure può considerarsi il suo primo servizio ufficiale ad alto livello nella Chiesa, grazie alla sua perizia scritturistica. Vi si percepisce tra l'altro una inconsueta interiorità contemplativa che resterà in seguito soffocata dai toni delle polemiche o di altra indagine nei testi sacri o preoccupazione di direzione spirituale. Ancora una volta perciò, papa Damaso riesce a strappargli un inedito volto di sé. Girolamo con finezza quasi esprime i propri disappunti come i propri entusiasmi, e lo fa con tono mite di discente più che di saggio docente interpellato, confessando aiuti ricevuti e limiti riconosciuti ${ }^{54}$.

Finalmente, si ha poi testimonianza di come papa Damaso si rivolgesse a Girolamo, trasferitosi già nell'Urbe: in una breve epistola gli chiede aiuto per l'interpretazione ufficiale del termine ebraico Osanna. Con la semplicità che doveva essergli naturale, esordisce:

„Damaso, vescovo, saluta Girolamo, figlio carissimo nel Signore”, e con altra nobile lode ne esorta rispettosamente il competente intervento: „La tua dilezione è nota per la potenza e l'arditezza dell'ingegno. Vedi dunque di eliminare le congetture e di dissipare gli equivoci, e fammi sapere chiaramente quale sia il senso autentico del passo, in ebraico. Di questo favore, come pure di molti altri, la nostra sollecitudine ti sarà grata, in Cristo Gesù"'55.

${ }^{54}$ Cf. $E p .18$ [A-B] , 4, 6, 7, 12, 15, 16, 18: CSEL 54, 78, 81, 84, 88, 93, 96, 99: „multo si quidem melius est vera rustice quam diserte falsa proferre; Ergo quaerimus ubi sit hoc incendium salutare. Nulli dubium, quin in sacris voluminibus, ex quorum lectione universa hominum vitia purgantur; in ambobus testamentis Trinitas praedicatur; non sunt, ut quidam putant, in scripturis verba simplicia; plurimum in his absconditum est. Aliud littera, aliud mysticus sermo significant; audivi ego hoc in loco non paruam Hebraei mei disputationem, cuius pauca ponam, ut sensum hominis advertas; Et quoniam usque ad finem capituli explanatio multiplex sequitur et excipientes iam inplevimus ceras, hucusque dictasse sufficiat, quia et oratio, quae non propriae manus stilo expolitur, cum per se inculta est, tum multo molestior fit, si taedium sui prolixitate congeminet, et oculorum dolore cruciati auribus tantum studemus et lingua; quantum autem ad mysticos intellectus, ille eis ignis mittitur, quem Hieremias ferre non poterat, qui, cum animae nostrae arcana penetravit, ita nos dissolvit, ita a veteri homine in novum excoquit, ut in illam vocem possimus erumpere: vivo autem iam non ego, sed gratia Dei, quae in me est (Gal 2, 20)".

55 Ep. 19, CSEL 54, 103-104: „Dilectissimo filio Hieronymo Damasus episcopus in domino salutem [...] dilection is tuae ardenti illo strenuitatis ingenio abscisis opinionibus ambiguitatibusque subplosis, quid se habeat apud Hebraeos, vivo sensu scribas, ut de hoc, sicut et de multis, tibi curae nostrae in Christo Iesu gratias referant". 
È il consolidamento di una collaborazione intensa, più ancora di un rapporto talmente profondo da mostrare quasi una gara di umiltà tra i due, anzi quasi di reciproca venerazione, che se non desta stupore per la signorilità d'animo del papa poeta, suscita invece meraviglia per l'inedito tratto del monaco dalmata che ne risulta.

Al biglietto di Damaso, Girolamo risponde ancora con un'esposizionedotta e riverente insieme, che ne valorizza la sua straordinaria competenza in lingua ebraica e termina con alcune dichiarazioni da doversi ritenere la magna charta dello studioso oppure il protocollo geronimiano del biblista:

„Sappia però la tua Beatitudine, che mai la noia dovrebbe invadere l'anima dello studioso, in ricerche di tal genere. Anche noi avremmo potuto facilmente inventare una menzogna, e con una parola tagliar la testa all'idra, come si è visto che hanno fatto gli altri. Ma è molto meglio faticare un po' per la verità e abituare il proprio orecchio ad una lingua straniera, piuttosto che proporre una soluzione fasulla, estranea al concetto di quella lingua" ${ }^{\text {"56. }}$.

L'asceta si schernisce di aver „dettato solo appunti sintetici, conformemente alla limitatezza del proprio ingegno" ${ }^{\prime 57}$, ma poi sembra voler giustificare la puntigliosa lezione impartita e forse scusarsi implicitamente con l'insigne amico per averlo probabilmente affaticato nel sottile procedimento scientifico condotto. Damaso, da parte sua, risulta soddisfatto del prezioso collaboratore, perché subito gli chiede una non meno semplice ermeneutica della parabola del figliol prodigo e Girolamo, appagato di tanta fiducia e stima da parte di quel vescovo di Roma suo protettore nell'Urbe, si impegna in una spiegazione esauriente e ricca di elementi morali, storici e spirituali. Dall'introduzione della lettera si comprende su quante domande lo avesse interpellato il caro amico senza escludere in ciò l'impiego di una probabile furbizia pedagogica per stimolare il genio del Dalmata, il quale infatti ossequioso risponde: „Il questionario della tua Beatitudine costituiva da solo una dissertazione" ${ }^{\text {.58 }}$.

Nel rapporto con Girolamo, papa Damaso investe l'evangelica scaltrezza cui sono invitati i figli della luce (cf. Lc 16,8), perché riesce con accortezza a spronare il suo segretario a produrre bellissimi testi esegetici. Veramente egli ne aveva bisogno, oppure l'interpella appositmente per indurlo a fissare su pergamena il frutto di tanti studi? L'intelligente opera di questo vescovo di Roma comunque, in tale direzione, non restò mai delusa. A nessun interlocu-

${ }^{56}$ Ep. 20, 5, CSEL 54, 110: „ceterum sciat beatitudo tua in istiusmodi disputationibus molestiam in legendo non debere subrepere, quia facile et nos potuimus aliquid ementiri, quod ex una voce solveret quaestionem, sicuti et ceteros fecisse monstravimus. Sed magis condecet ob veritatem laborare paulisper et peregrino aurem adcommodare sermoni, quam de aliena lingua fictam ferre sententiam".

57 Ibidem: „Haec interim iuxta mediocritatem sensus mei breviter strictimque dictavi”.

${ }^{58}$ Ep. 21, 1, CSEL 54, 111: „Beatitudini tuae interrogatio disputatio fuit”. 
tore l'irascibile monaco ha mai risposto nei seguenti termini: „Porre così le domande è già aver aperto la via alla risposta. E si sa che 'chi interroga con sapienza dev'essere ritenuto saggio' $(\operatorname{Pr} 17,28)$ "59.

Dalla richiesta di Damaso, in ogni caso, scaturirà un'altra lezione elevata di Girolamo. Nel testo, da rilevare, è il confidenziale $t u$ con cui gli si rivolge durante l'esposizione, quasi fosse in familiare conversazione, rapito com'è nel suo compito per l'amico presente al suo cuore. Alla profondità delle intuizioni spirituali si aggiungono quindi osservazioni fatte quasi a se stesso, o riflessioni sui propri problemi, come il lamentare nuovamente quel male agli occhi che da tempo lo affligge:

„Non ne dubito: ti sembrerà certamente rozzo lo stile di questo povero uomo; ma sovente ho sperimentato che è impossibile abbellire il proprio linguaggio senza un lungo lavoro di lima, fatto di proprio pugno. Abbi compassione dei miei occhi malati, perdona a chi è costretto a dettare. Tanto più che nelle scienze ecclesiastiche, non si bada alla raffinatezza dell'espressione, ma al contenuto. È con il pane che ci si tiene in vita, non con legumi" ${ }^{\prime 60}$.

E forse per gli occhi malati, forse per l'impegno con le nobildonne ascete dell'Aventino, oppure semplicemente per per la sua discrezione nella posizione di segretario del papa, insieme all'invincibile passione per le Sacre Scritture, Girolamo pare essersi poi impigrito quanto a scrivere. Damaso, infatti, sembra quasi doverlo riportare con forza a questo compito che garantisce a molti i benefici del suo studio personale. Si rivolge quindi ancora al „al carissimo figlio" per sollecitarlo con precise domande esegetiche.

Dall'epistola del vescovo di Roma risulta che il Dalmata gli ha addirittura promesso di lavorare di notte per lui, in caso di richiesta di spiegazioni bibliche e Damaso accetta ora l'offerta. Tra le righe di tono scherzoso si comprende un rapporto ancora più stretto quanto più sono accresciute la stima, il rispetto, la confidenza e la reciproca conoscenza. Si comprende pure quanto, dietro l'apparente interesse immediato dell'anziano papa, vi sia soprattutto il desiderio di far scrivere Girolamo per possederne opere valide da diffondere. Infatti, dichiara che „con tutta avidità" ha „letto e fatto ricopiare" precedenti lettere addirittura dettate dal Dalmata dal deserto di Calcide ${ }^{61}$.

59 Ibidem: „sic quaesisse quaerenda viam est dedisse quaesitis. Sapienter quippe interroganti sapientia reputabitur". Soltanto con le donne sue collaboratrici, e in specifico Marcella, Paola ed Eustochio, il Leone dalmata fu così disponibile. Lo stesso Agostino ebbe a faticare non poco per entrare in eguale relazione con lui su questo piano, cf. $E p .110,5$, CSEL 55, 361.

60 Ep. 21, 42, CSEL 54, 142: „Non ambigo, quin inculta tibi nostrae parvitatis videatur oratio; sed saepe causatus sum excoli non posse sermonem, nisi quem propria manus limanuerit. Itaque ignosce dolentibus oculis, id est ignosce dictanti, maxime cum in ecclesiasticis rebus non quaerantur verba, sed sensus, id est panibus sit vita sustentanda, non siliquis".

${ }^{61}$ Cf. Ep. 35, 1, CSEL 54, 265: „[epistulas] quas in heremo aliquando dictaveras quasque tota aviditate legi atque descripsit". 
Con ironia Damaso si era permesso di pungere l'orgoglio di Girolamo iniziando la lettera come segue:

„Ho l'impressione che da troppo tempo passi le tue giornate piuttosto nella lettura che non a scrivere; ma ho trovato il modo di svegliarti dal sonno mandandoti alcuni problemi. Non che tu non debba anche leggere..., ma se scrivessi avremmo anche delle opere, come frutto della lettura"62.

Per la prima volta troviamo tanta sincerità aperta nel carteggio rimasto del beatissimo amico Damaso a Girolamo, oltre che tanta decisa confidenzialità come unica autorità influente certamente, e positivamente, sulla disponibilità del monaco ad accontentarlo. Non solo, ma Damaso si prende licenza persino di indicare al Leone dalmata come desidera che esegua un lavoro che diviene un ordine perentorio, non un invito. Leggiamo accenti ben diversi e lontani da quelli di epistole di anni precedenti:

„Al lavoro, dunque! e dammi spiegazione di quanto ti sottopongo. Ma sii moderato in due sensi: che le risposte siano esaurienti, ma non eccedano la brevità richiesta da una lettera"63.

Con riverenza e sollecitudine, l'obbedienza del monaco è immediata, unitamente alla richiesta di perdono

„tanto della fretta che del ritardo: della fretta, perché in una sola breve veglia ho voluto dettare un argomento che avrebbe richiesto più giorni; del ritardo, perché non ho risposto subito alla tua richiesta, occupato com'ero in altro lavoro"64.

Che Damaso sia riuscito a colpire l'amico anche nell'orgoglio, lo si capisce dalla velata reazione di Girolamo là dove dichiara, anch'egli in tutta sincerità:

„Ho tra mano - a dirtela francamente - un volume di Didimo sullo Spirito Santo. Desidero tradurlo e dedicartelo; così non pensi che me ne sto a dormire, dato che per te leggere senza scrivere è dormire" 65 .

Il Leone dalmata si è svegliato e lo ritroviamo in pieno vigore intellettivo, passionale, ironico e arguto. Non ha avuto remore già a dichiare palesemente senza più ombra di soggezione verso quel vescovo di Roma a lui così unito:

62 Ibidem: „Dormientem te et longo iam tempore legentem potius quam scribentem quaestiunculis ad te missis excitare disposui, non quo et legere non debeas [...], sed quo lectionis fructus sit iste, si scribas".

${ }^{63}$ Ep. 35, 2, CSEL 54, 266: „Accingere igitur et mihi, quae subiecta sunt, dissere seruans utrobique moderamen, ut nec proposita solutionem desiderent nec epistulae brevitatem".

${ }^{64} E p .36,1$, CSEL 54, 269: ,adnexui veniam postulans et festinationis pariter et morarum: festinationis, qui ad unam lucubratiunculam dictare volui rem dierum; tarditatis, quia alio opere detentus non statim ad interrogata rescripsi".

65 Ibidem: „et ut verius loquar, Didymi de Spiritu Sancto librum in manibus habeo, quem translatum tibi cupio dedicare, ne me aestimes tantummodo dormitare, qui lectionem sine stilo somnum putas". 
„Ma tu, ancora ieri, hai detto al diacono, che mi hai mandato, di riferirmi che stai aspettando quella che tu pensi una lettera (io la considero piuttosto un trattato!) $\mathrm{e}$ che desideri una breve risposta a quelle domande che, prese anche singolarmente, richiederebbero un poderoso volume"

Questo simpatico colloquio scritto, e in tal modo fortunatamente rimastoci tra i due amici, è una finestra aperta sul loro rapporto di reciproco aiuto ciascuno al carisma dell'altro col solo timore di deluderlo o di non valorizzarlo pienamente. I ruoli scompaiono: restano due uomini l'uno di fronte all'altro, indefessi nel proprio impegno di dare tutto di sé e il meglio della loro esperienza spirituale e cristiana a gloria di Dio e a edificazione del prossimo. Nessun ripiegamento, egoismo o vanagloria in loro: soltanto una profonda umiltà capace di correzione fraterna con scherzo amorevole e di visione non inquinata dei doni dell'altro.

Ancora una volta, comunque, la mite forza del poeta spagnolo ha vinto l'impetuosità del biblista dalmata, confermando l'integrazione perfetta di due personalità in sintonia e l'unicità, per quanto almeno concerne Girolamo, di una relazione umana costruttiva per tutte le sue qualità interiori, spirituali e culturali. Damaso avrà così il merito non solo di avergli commissionato la Vulgata, bensì di essere riuscito pure a fargli esprimere quella carica di umanità e di elevazione mistica che mai più il monaco di Betlemme, in seguito oppresso da lotte e prove d'ogni tipo, saprà esprimere tanto intensamente.

2. Bonifacio. Diverso è lo stile con cui Girolamo si relaziona al „beatissimo papa Bonifacio", pur con analogia di confidenza e rispetto avuti con papa Damaso. L'unico documento testimone di ciò è degli ultimi mesi della vita del monaco dalmata e, secondo quanto vi si legge, l'elezione di questo amico alla Cattedra di Pietro è la sola notizia buona che gli giunge in quel periodo e che l'allevia dal dolore inconsolabile per la perdita della figlia spirituale Eustochio.

Il tono è amichevole, dettato da un intimo „tu”: „Non ti so dire la gioia che ho provato nel venire a sapere della tua elezione al pontificato". Parla quindi di un legame da tempo instaurato sul „reciproco affetto”, ma proprio questo, per paradosso, costituisce il momento di giubilo che lo porta per un po' fuori dal suo dolore, poiché dichiara: „Ho l'anima così fusa in uno con la tua che non s'accorge neppure dello sfacelo del corpo", e pure nell'ombra dello stesso perché quel legame con Bonifacio gli rinnova quanto l'abbatte, cioé „la scomparsa della santa e venerabile vergine di Cristo Eustochio"67. Infatti, Bonifacio

${ }^{66} E p .36,1$, CSEL 54, 268: „verum, quia heri diacono ad me misso, ut tu putas, epistulam, ut ego sentio, commentarium te expectare dixisti brevem responsionem ad ea desiderans, quae singula magnorum voluminum indigent".

${ }^{67}$ Ep. 153, CSEL 56, 365-366: „Beatissimo papae Bonifatio Hieronymus. Quantum gaudii super ordinatione pontificatus tui sancto Innocentio presbytero et nuntium et litteras tuae beati- 
era parente di questa discepola prediletta di Girolamo, il quale subito pensa di riflesso nel suo lutto:

„T'immagini come [Eustochio] avrebbe saltato dalla contentezza se avesse meritato di conoscere, ancora viva, questa notizia? Immagini quali preghiere di ringraziamento avrebbe rivolto alla clemenza di Cristo al sapere che un santo e venerabile suo parente è stato insediato sulla cattedra apostolica"68.

All'insigne amico lo lega pure la comune educazione impartita a Paola, figlia di Tossozio e di Leta. Santa Paola era madre di Tossozio e di Eustochio; la loro nipotina Paola era dunque passata dall'insegnamento di Bonifacio, loro parente, alla direzione monastica di Girolamo, loro guida spirituale ${ }^{69}$. A queste note di intesa formativa e pastorale tra Girolamo e Bonifacio, non mancano quelle del comune servizio alla verità e alla fede, nonostante si comprenda quanto ormai a Girolamo manchino persino le forze di scrivere ${ }^{70}$.

Soltanto un post-scriptum, infatti, è di suo pugno e suona come un testamento spirituale al neoeletto vescovo di Roma. In poche righe si concentra tutta la combattiva personalità dell'asceta di Betlemme che incita l'amico a nette prese di posizione in difesa dell'ortodossia, dicendogli:

„Gli eretici si accorgano che tu sei un nemico della loro perfidia, e che ti odino; così i cattolici ti ameranno di più" 71 .

A parole forti, seguono verbi imperativi più che esortativi:

„Da' corso ed esecuzione alle decisioni dei tuoi predecessori, e non permettere che abbiano titolo di vescovo i patroni e i complici degli eretici"72.

tudinis perferente susceperim, ambigere non potest tua reverentia, cum olim mutuo iungamur affectu et ante coeperimus nos amare quam nosse interiorque homo ita sibi coniunctus sit, ut exterioris hominis damna non sentiat. Haec sola res dolorem nostrum super dormitione sanctae ac venerabilis virginis Christi Eustochiae mitigavit, nisi quod et in hoc tristitia non minor sit, quod tanto nobiscum priuata sit gaudio".

68 Ibidem, CSEL 56, 366: „quo enim illa, si hoc in corpore constituta audire meruisset, gestisset gaudio, quibus precibus et gratiarum actione Christi clementiam flagitasset, quod sanctum ac venerabilem parentem suum apostolicae cathedrae successorem esse didicisset!".

${ }^{69}$ Cf. ibidem: ,infans Paula, quae in tuis nutrita est manibus, quasi pignus sanctae ac venerabilis memoriae Laetae nostris est inposita ceruicibus", cf. Mirri, La dolcezza nella lotta, p. 108-111.

${ }^{70}$ Cf. ibidem: „Obsecro reverentiam tuam, ut quasi ad tuos semper adscribas et nos proprie super profectu et honore reverentiae [tuae] gaudere cognoscas. Certe sanctus ac venerabilis Innocentius presbyter beatitudini tuae poterit indicare, quantum in ipso maerore gaudii ceperimus et quomodo, si fieri posset, tuis cuperemus haerere conplexibus".

${ }^{71}$ Ibidem: „Propria manu quod scribo, beatitudini tuae scribo. Sentiant heretici inimicum te esse perfidiei et oderint, ut a catholicis plus ameris".

72 Ibidem: „Et executor atque conpletor sis sententiae praecessorum tuorum nec patiaris in episcopali nomine hereticorum patronos atque consortes". Papa Bonifacio non fu però poi così energico contro gli eretici pelagiani. Alcune sue lettere si trovano in PL 20, 750-783. 
A differenza che con papa Damaso, con il quale Girolamo era in uno stretto rapporto di collaborazione e di intesa anche su tranquilli orizzonti spirituali e culturali, con papa Bonifacio si manifesta un relazione fondata piuttosto sulla solidarietà della battaglia all'eresia e di diversità di carattere nella reazione contro i nemici della fede e della Chiesa, lotta che l'ha sfibrato e che, per l'età e la malattia, non è più in grado di affronare apertamente e direttamente: passa così il testimone al fidato vescovo di Roma, dal quale presagisce, forse, di congedarsi per sempre, dopo non avergli negato quell'ultimo suo aiuto in consiglio $^{73}$.

\section{SUCCESSORI DI PIETRO „DEFENSORES FIDEI”}

La controversia origenista e quella pelagiana videro Girolamo impegnato come acerrimo nemico dell'eresia con le sue opere di argomento biblico e con la sua arguta satira contro gli eretici. Di due successori di Pietro, resta una bella testimonianza del santo Dottore nella lettera che egli indirizza alla vergine Demetriade, alla quale raccomanda con accorate espressioni che riflettono i problemi del tempo, di attenersi strettamente ai loro insegnamenti:

„Stavo quasi per dimenticare la cosa più importante. Quando eri ancora bambina e al governo della Chiesa di Roma sedeva papa Anastasio, di santa e felice memoria, la perfida tempesta dell'eresia che si era scatenata in Oriente fece di tutto per macchiare e distruggere la semplicità di quella fede che era stata lodata dalla voce stessa degli Apostoli. Ma quell'uomo, ricchissimo della sua povertà e animato da zelo apostolico, colpì subito la testa velenosa dell'idra e ne tenne a freno la bocca sibilante. Tuttavia, siccome temo, o meglio so per sentito dire che l'eresia alligna ancora in alcuni, e che i suoi polloni continuano a germogliare, spinto da un sentimento di affettuosa carità, penso doverti dare questo preavviso: custodisci la fede del santo Innocenzo, figlio e successore di Anastasio, e non dare ascolto a una dottrina esotica, per quanto prudente e ingegnosa possa sembrarti"74.

${ }^{73}$ Della situazione ormai drammatica di Girolamo negli ultimi mesi di vita è testimone pure il termine della lettera indirizzata a Ripario, eroe nella lotta antipelagiana. Girolamo descrive con le seguenti parole la sua condizione: $E p .152$, CSEL 65, 365: „quod autem ad scribendum cohortaris, grave asello vetulo imponis onus. Nos enim et acumen ingenii et vires corporis penitus deserverunt, quas adsidua morborum debilitate perdidimus. Incolumem te et mei memorem Christi, Dei nostri, tueatur clementia, domne vere sancte et multum desiderande frater".

${ }^{74}$ Ep. 130,16, CSEL 56, 196: „Paene praeterii, quod vel praecipuum est. Dum esses parvula et sanctae ac beatae memoriae Anastasius episcopus Romanam regeret ecclesiam, de Orientis partibus hereticorum saeva tempestas simplicitatem fidei, quae apostoli voce laudata est, pollvere et labefactare conata est. Sed vir ditissime paupertatis et apostolicae sollicitudinis statim noxium perculit caput et sibilantia hydra ora conpescuit. Et quia vereor, immo rumore cognovi in quibusquam adhuc vivere et pullulare venerata plantaria, illud te pio caritatis affectu praemonendam puto, ut sancti Innocentii, qui apostolicae cathedrae et supra dicti viri successor et filius est, teneas fidem nec peregrinam, quamvis tibi prudens callidaque videatur, doctrinam recipias". Per Demetriade, cf. Mirri, La dolcezza nella lotta, p. 95-96. 
1. Anastasio. Purtroppo, il breve pontificato di questo vescovo romano, appena di un biennio, non consentì rapporti personali con l'eremita di Betlemme, fosse solo anche epistolarmente. Girolamo, che era stato grande estimatore di Origene, si trovò ad un certo momento nella necessità di prendere una posizione contro quel maestro di commento biblico. Lo fece a favore di quanti guardavano a lui per la verità dottrinale della Chiesa, anche se, probabilmente, certe dispute dogmatiche, per lui esegeta, continuarono ad apparire piuttosto come „capricci dialettici" che come serie teorie.

Nel suo epistolario esiste un carteggio che non lo concerne direttamente in qualità di mittente o di destinatario, ma che è senz'altro passato per le sue mani favorendo le sue decisioni. I documenti infatti riguardano la decisione del vescovo di Roma sulla complessa diatriba origeniana. Si può a ragione pensare che, sebbene l'epistola di Anastasio sia indirizzata a Simpliciano di Milano perché nell'Italia settentrionale si conosca la condanna dell'origenismo pronunciata in modo eccellente e chiaro dalla lettera sinodale di Teofilo ${ }^{75} \mathrm{di}$ Alessandria d'Egitto, ratificata pure dall'autorità imperiale in Oriente, abbia confermato Girolamo nella sua fede e nella sua unità con il successore di Pietro.

Nel testo di papa Anastasio gli scritti di Origene sono messi all'indice. Viene elogiato l'operato di Teofilo, attivissimo nell'estirpare l'errore sia corrispondendo con i confratelli di Palestina e Cipro, di cui si ha riscontro nelle lettere di risposta ${ }^{76}$, al fine di convocare un sinodo sulla questione, sia accertando la professione di fede confessata negli influenti monasteri di Nitra ${ }^{77}$.

Anastasio elogia il „pastore accorto" che „dimostra grande premura per il suo gregge e lo sa sorvegliare". Con altra immagine parla di nave nel mare in burrasca e salvata dal pilota tutto teso ad evitare con ogni ansia e perizia che essa si infranga sugli scogli. Allo stesso modo Teofilo, in quell'ora difficile per la fede, „vigila senza un attimo di riposo per il bene della salvezza”, evitando al popolo di Dio di cadere in "madornali bestemmie"78.

Questo modo di esprimersi non può che essere piaciuto a Girolamo, il quale era in contatto epistolare con Teofilo ${ }^{79}$ e quindi informato delle decisioni e del pensiero di papa Anastasio. Inviando a Roma la traduzione latina della

${ }^{75}$ Cf. Ep. 92, CSEL 55, 147-155.

${ }^{76}$ Cf. Ep. 93 e 94, CSEL 55, 155-157.

${ }^{77}$ Cf. $E_{p} .95,1-2, \operatorname{CSEL} 55,158:$,[...] ne quis contra praeceptum legat haec, quae diximus, damnavimus".

${ }^{78}$ Ep. 95, 1, CSEL 55, 157-158: „Grandem sollicitudinem atque excubias super gregem suum pastor diligens habere adprobatur [...] magister attentus navis hora tempestatis e curis et periculo magnam patitur animi iactationem, ne procellis atque asperrimis fluctibus navis elidatur in saxa. Pari animo vir sanctus et honorabilis Theophilus, frater et coepiscopus noster, circa salutis commoda non desinit vigilare, ne Dei populus per diversas ecclesias Origenem legendo in magnas incurrat blasphemias".

${ }^{79}$ Cf. Ep. 86, 87, 88, 89, CSEL 55,138-143; Ep. 96, CSEL 55, 159-181. 
lettera pasquale di Teofilo (Ep. 118), Girolamo scrive agli amici Pammachio e Marcella le proprie riseve contro gli originisti e aggiunge:

„Si sa, d'altronde (dato che la notizia è giunta ovunque), che lo stesso beato papa Anastasio ha dato addosso agli eretici, che stavano nascosti nelle loro tane, con uno zelo non inferiore. Egli, infatti, la pensa allo stesso modo, e le sue lettere devono considerarsi come un documento che l'Occidente ha condannato quello che ha condannato l'Oriente. Gli auguriamo molti anni di vita, affinché possano morire del tutto i polloni redivivi di questa eresia che per tanto tempo sono insecchiti grazie alla sua attività ${ }^{\prime 80}$.

Purtroppo, invece, le cose non andarono così: Anastasio morì presto, il 19 dicembre 402. Con questo successore di Pietro, dunque, il santo Dottore si trovò a collaborare in modo indiretto lottando contro gli eretici, appoggiando le idee e diffondendo la dottrina che la sede di Roma aveva siglato con propria autorità, anche tramite l'encomio d'un pastore con cui Girolamo si sentiva unito per fede, stima e ministero. Di lui, infatti, scriverà alla vergine Principia:

\begin{abstract}
„Nel frattempo salì al trono pontificio un uomo illustre: Anastasio; ma Roma non meritò di avere a lungo un vescovo come lui, perché non doveva verificarsi che il mondo si vedesse troncare il capo durante il suo pontificato. Anzi, fu portato via e trasferito altrove, perché non tentasse di piegare con le sue preghiere la condanna già fissata" ${ }^{\prime \prime}$.
\end{abstract}

2. Innocenzo. Le sofferenze di questo successore di Pietro hanno avuto un loro culmine nel tragico sacco di Roma perpetrato da Alarico nel 410, ma non cessarono a motivo di altri conflitti e saccheggi compiuti da cristiani eretici anche nelle terre d'Oriente, dove le lotte infuriavano. Girolamo da Betlemme aveva continuato a collaborare quasi direttamente con il papa in questa battaglia contro gli errori in materia di fede tramite la dotta Marcella, competente in Sacra Scrittura quanto il maestro, che a lei rimandava con fiducia messi del vescovo di Roma bisognosi di delucidazioni in materia ${ }^{82}$.

Questo ininterrotto cooperare a servizio della fede, anche se mediato da persona tanto stimata da Girolamo, rese i vincoli con la sede romana talmente

${ }^{80} E_{p .}$ 97, 4, CSEL 55, 184: „quoque papam Anastasium eodem fervore, quia eodem est spiritu, latitantes in foveis suis hereticos persecutum eiusque litterae doceant damnatum in occidente, quod in oriente damnatum est. Cui multos inprecamur annos, ut hereseos rediviva plantaria per illius studium longo tempore arefacta moriantur". Per Anastasio, cf. F. Cavallera, Saint Jérôme. Sa vie et son oeuvre, Louvain 1922, I, 255-262; II, 38-43.

${ }^{81}$ Ep. 126, 10, CSEL 56,153: „non multum tempus in medio, succedit in pontificatum vir insignis Anastasius, quem diu Roma habere non meruit, ne orbis caput sub tali episcopo truncaretur; immo idcirco raptus atque translatus est, ne semel latam sententiam precibus suis flectere conaretur". Di Anastasio, Girolamo fa riferimento anche nell'Ep. 126, 1, CSEL 56, 143. Per Principia, cf. Mirri, La dolcezza nella lotta, p. 94.

82 Cf. Mirri, La dolcezza nella lotta, p. 132-138. 
stretti da indurre l'asceta dalmata a rivolgersi senza esitazione ad Innocenzo I quando i pelagiani attuarono razzie e distruzioni nei suoi monasteri di $\mathrm{Be}-$ tlemme, mettendo a rischio la sua stessa vita.

Troviamo così una breve corrispondenza del 416 che coinvolge papa Innocenzo, il vescovo di Cartagine Aurelio cui Girolamo si rivolse per far giungere al successore di Pietro le drammatiche notizie, e Giovanni, il vescovo di Gerusalemme, duramente richiamato poi dal papa al suo dovere di pastore e reso responsabile dell'accaduto al gregge sotto sua giurisdizione. Questo spiega perché Girolamo, per far giungere al papa il suo grido di aiuto da Betlemme, si sia rivolto ad Aurelio di Cartagine, vescovo di sua fiducia.

Innocenzo, a sua volta, scrive ad Aurelio con parole commoventi verso il monaco dalmata, appellandolo „collega di sacerdozio” e affermando: „Ho un senso di compassione per quel membro del nostro gregge" 83 . Dichiara, inoltre, di aver già attuato drastiche decisioni. Attraverso il „carissimo fratello Aurelio", uomo di fiducia sia per lui che per l'asceta di Betlemme, il papa fa pervenire quindi a quest'ultimo una sua lettera personale in cui scrive:

„Le tue grida di dolore hanno scosso il mio amore... mi congratulo con te per la tua fedeltà e perseveranza" ${ }^{\prime 4}$.

Girolamo nella sua lettera si era schernito dicendo che chiunque si sarebbe lasciato calunniare e minacciare per difendere la verità ${ }^{85}$. In questa umiltà, il papa esalta nel santo Dottore una non comune forza che che l'ha esposto al maggior pericolo accaduto. Parla, infatti, di „scenario di male" e di intervento „dell'Autorità della Sede Apostolica per reprimere ogni empietà", tanto gravemente si presenta la situazione. Ma la confidenza gli permette di avanzare un appunto delicato:

„Ma, contro chi scagliarmi? Non vi ho trovato nominata una sola persona e nessuno è incolpato di un delitto, per quanto poco motivato" ${ }^{\text {"86 }}$.

Girolamo non farà nomi, ma l'insigne amico vescovo di Roma comprende la sua discrezione e pure le responsabilità del vescovo di Gerusalemme, l'unico contro il quale, probabilmente, ha preso il duro provvedimento annunciato. Gli invia, infatti, una lettera severissima, in cui, tra l'altro, persino dice:

${ }^{83}$ Ep. 135, CSEL 56, 263: „Piissimum iter ad nos perveniendi tuas affectiones bene conpresbyter noster credidit Hieronymus. Conpatimur gregis nostri membri et, quod faciendum duximus vel facere potuimus, sumus velociter executi".

${ }^{84}$ Ep. 136, CSEL 56, 263-264: „dolor et gemitusque tuus ita viscera quatit nostra [...] primum constantia tuae adloquor fidem".

${ }^{85}$ Cf. ibidem, CSEL 56, 264: ,pro veritate quisque iniura aut, ut dicis, periculo percelletur".

${ }^{86}$ Ibidem: „item excitati tanta malorum scena arripere auctoritatem sedis apostolicae ad omne comprimendum nefas festinavimus, sed, in quem insurgeremus, nec nomine appellatum legimus nec criminis aliqua ratione taxatum". 
„Il potere che il diavolo ha preso su di te e sui tuoi non riesce a scuotere proprio per niente quella tua risaputa pietà di vescovo? Su di te, dico! Perché il fatto che nella tua Chiesa sia stato commesso un misfatto così esecrabile è senz'altro un capo d'accusa contro la tua carica vescovile" ${ }^{\prime 87}$.

Segue l'accusa di omissione di misure di difesa e di carità, oltre la minaccia di ricorso a sanzioni di diritto ecclesiastico per non aver difeso „la causa degli oppressi ${ }^{88}$.

Innocenzo manifesta pertanto fermezza e temperamento, e questo deve essere stato un punto d'incontro tra lui e Girolamo, seppure a distanza. L'intesa tra $\mathrm{i}$ due non aveva ombre in condivisione di stile e di strategie contro gli eretici, e quando questi si approfitteranno di assalire gli insediamenti monastici di Betlemme, Innocenzo da Roma non può che restare colpito da tanta violenza che la questione assume. „Mi limito a manifestarti il mio dolore”, scrive all'asceta dalmata, ma se le denunce saranno più precise, dichiara pure:

"Ti designerò dei giudici competenti, oppure, se c'è qualcosa di più urgente e di più pressante che mi sia possibile fare, non perderò un attimo di tempo, figlio mio carissimo" $"$.

Sono toni di solidarietà struggenti, da un altro uomo ormai giunto a conclusione delle sue fatiche ed al quale, come all'amico biblista, queste sofferenze hanno certamente contribuito ad abbreviare il pellegrinaggio terreno. Pochi mesi dopo, nel 417, Innocenzo morirà. Girolamo lo seguirà nella vita eterna circa due anni più tardi. Per designare l'intesa di entrambi vale la parola dell'Apostolo nella quale, insieme fino all'ultimo, si sono identificati: „Ho combattuto la buona battaglia, ho terminato la mia corsa, ho conservato la fede" $(2 \operatorname{Tm} 4,7)$.

\section{$* * *$}

Parlando di Pietro e di alcuni suoi primi successori tra i Viri Illustres Clemente, Vittore e Cornelio - Girolamo sembra preoccupato di segnalare

${ }^{87}$ Ep. 137, CSEL 56, 264-265: ,nihil monuet pietatem illam sacerdotii tui de tanta diaboli in te atque in tuos potestate admissa? In te, inquam;prorsus enim sacerdotis gravitatem condemnat tantum nefas in ecclesia fuisse completum".

${ }^{88}$ Cf. ibidem: „Direptiones, caedes, incendia, omne facinus extremae dementiae generosissimae sanctae virgines Eustochium et Paula deploraverunt in locis ecclesiae suae perpetrasse diabolum [...] amicem gregem domini et tales agnas incendio, armis et persecutionibus nudas, debiles post suorum caedes et mortes vix vivere audivimus [...] ubi provisiones tuae? [...] vide, frater, antiqui hostis insidias et spiritu boni rectoris pervigila, ut haec, quae ad nos opinione vel retundantur, ne ius ecclesiasticum de labefactatis causas eum, qui non defenderit, praestare conpellat".

${ }^{89} E$. 136, CSEL 56, 264: „quod ergo possumus, condulemus. Si deposueris autem apertam manifestamque in homines aliquos accusationem, aut iudices conpetentes tribuam aut, si aliquid urgentibus sollicitiusque nobis fieri potest, non retardabo, fili dilectissime". 
l'anello di successione apostolica che diviene una connessione di fede. Pietro è il difensore dell'annuncio cristiano contro l'impostura di Simon mago e l'illustre martire di Nerone, ma innanzi tutto è il testimone della risurrezione e l'universale riferimento dei credenti: è la Roccia contro la quale si infrangono i marosi degli errori che travisano l'autentica dottrina cattolica ed è il fondamento della Tradizione.

Clemente è l'uomo di pace che spende il proprio ministero per l'unità dei fedeli ed esercita con umiltà il servizio di guida del popolo di Dio, illuminato dalla ispirazione della divina sapienza che lo aiuta nel faticoso compito ereditato nella sede dell'Urbe. Vittore è il vescovo di Roma delle decisioni liturgiche importanti per nutrire la vita spirituale del popolo di Dio. Egli, inoltre, ufficializza nella Chiesa la lingua latina rafforzando così, agli occhi del Dalmata, l'autorità della Cattedra di Pietro nella cristianità. Cornelio è il papa in lotta contro nemici interni ed esterni alla Chiesa, ma in cui si manifesta anche la moderazione, l'equilibrio, il primato della misericordia unitamente all'energica apologia della verità e alla pratica organizzazione della comunità ecclesiale. Il suo martirio sancisce come quello di Pietro, la Roccia, la solidità di un ministero atto a confermare nella fede e nella carità i fratelli.

Dunque, in questi medaglioni, Girolamo dà importanza al vescovo di Roma in quanto lo considera l'anello diretto con il kerigma della Chiesa primitiva, il testimone e il difensore della fede fino al martirio, se necessario, e l'uomo che assicura l'unità e la verità nella dottrina, nella cultura e nella capacità di governo della comunità cristiana.

Due papi suoi beatissimi amici, Damaso e Bonifacio, furono da lui stimati per queste qualità. In particolare, il rapporto fu profondo e documentato con il primo, del quale era divenuto stretto collaboratore. L'espressione più felice in riferimento a Damaso è quella che Girolamo gli invia dal deserto di Calcide per sapere quale sia la vera professione di fede cattolica, tra le molte incertezze terminologiche greche, dichiarando che solo presso „la Cattedra di Pietro si trova quella fede che la bocca di un Apostolo ha esaltato" e che, quindi, solo lì „si conserva intatta l'eredità dei padri" ${ }^{\circ}$. Riconosce al vescovo di Roma il ministero di servizio all'unità della Chiesa e in Damaso esalta la qualità poetica e spirituale, oltre che l'umiltà nella sapiente ricerca di conoscenze bibliche. Per quest'ultima, il monaco esegeta gli si metterà a disposizione con tutta la sua competenza in materia. L'obbedienza di Girolamo alle richieste di Damaso è generosa, immediata e commovente per la venerazione con cui si prodiga in favore dell'insigne amico.

Il rapporto con Bonifacio fu quello del ricordo di un'antica amicizia, coltivata per comune impegno formativo verso i componenti d'una famiglia legata alla storia personale di entrambi. Ci sono da parte del Dalmata i toni dell'addio

${ }^{90}$ Ep. 15, 1, CSEL 54, 62-63. 
alla vita terrena, ormai consumata, ma con la gioia di passare il testimone a chi per dovere di ministero dovrà proseguire la lotta contro gli eretici.

$\mathrm{E}$ i successori di Pietro defensores fidei contro origenisti e pelagiani già incrociatisi con Girolamo erano stati in specifico due: Anastasio, attraverso la mediazione di Teofilo d'Egitto, e Innocenzo, a cui l'asceta di Betlemme si appellò direttamente ricevendone solidarietà nel momento più tragico di persecuzione. Che fossero energici o meno in questa salvaguardia dell'ortodossia, prerogativa principale del pastore secondo Girolamo e in particolare di quello romano, il rispetto e la collaborazione del santo Dottore non verranno meno fino all'ultimo nei riguardi di chi si succedette sulla Cattedra di Pietro. Esempio ne sono le parole che egli spese, a termine della sua esistenza terrena ancora, per giustificare la debole risolutezza di Bonifacio contro i pelagiani: fragile non ne uscì il papa, dal suo giudizio netto, bensì quegli eretici considerati infine irrecuperabili!

A Donato che lamentava l'inadeguatezza dell'intervento del neoletto vescovo di Roma, il Dalmata scrisse infatti con bella considerazione:

„La misericordia di Cristo, tuttavia, mi fa credere che il mio santo signore e venerabile vescovo Bonifacio li sradichi con il soffio di Cristo. Dobbiamo scusarlo se, per ora, che si trova all'inizio della sua attività di Pontefice, offre loro una mano caritatevole e se cerca di salvarli per la via della clemenza e della mansuetudine. Non guariranno mai, comunque" $"$.

Allo stesso modo, per papa Damaso, tutto l'epistolario geronimiano è costellato di riferimenti di encomio ${ }^{92}$.

Dunque, Girolamo si conferma „vir maxime catholicus"93 non solo per essere rimasto nella verità della fede fino alla morte pur tra molte eresie, ma anche perché la sua unione con il successore di Pietro costituì una cementata fedeltà alla certezza della verità e una garanzia dell'unità alla quale tenne più di ogni rispetto umano e per la quale collaborò spendendosi senza riserva fino allo stremo delle sue forze ed investendo tutto il suo non comune genio umano e spirituale, tutta la sua passione culturale e intellettuale, tutto il suo impegno di vero cristiano e di santo monaco.

${ }^{91}$ Ep. 154 1, CSEL 56, 367: „tamen credimus in Christi misericordiam, quod dominus meus sanctus et venerabilis episcopus Bonifatius eradicet eos spiritu Christi, cui debemus ignoscere, si in principiis suis offert caritatem et per clementiam suam et mansuetudinem seruare conatur, qui tamen numquam curandi sunt".

92 Cf. Ep. 17, 2, CSEL 54, 71; Ep. 22, 22, CSEL 54, 175; Ep. 36, praef., CSEL 45, 268; Ep. 45, 3, CSEL 54, 325; Ep. 49, 18, CSEL 54, 382; Ep. 80, 1, CSEL 55, 108; Ep. 84, 2, CSEL 55, 121; Ep. 123 , 9, CSEL 56, 82.

93 Sulpicius Severus, Dialogus I 7, CSEL 1, 159: „PPostumianus ait] quod Hieronymus, vir maxime catholicus et sacrae legis peritissimus, Origenem secutus primo tempore putabatur". 


\title{
HIERONIM A BISKUPI RZYMU - WZAJEMNE POSZANOWANIE I WSPÓŁPRACA
}

\author{
(Streszczenie)
}

Mówiąc w De viris illustribus ośw. Piotrze i o niektórych jego pierwszych następcach - Klemensie, Wiktorze i Korneliuszu - Hieronim wydaje się być wielce zatroskany, by dokładnie wskazać sukcesję apostolską, która jest gwarancją wiary. Piotr jest obrońcą przesłania chrześcijańskiego przeciwko fałszowi Szymona Maga, lecz przede wszystkim jest świadkiem zmartwychwstania i punktem odniesienia dla wszystkich wierzących - jest Skałą, o którą rozbijają się błędy przeciwne nauce katolickiej i Tradycji.

Klemens jest człowiekiem wprowadzającym pokój, który poświęca swój urząd jedności wiernych i z pokorą wypełnia posługę przewodnika ludu Bożego. Wiktor jest biskupem Rzymu, który podejmuje ważne decyzje odnośnie do liturgii, aby w ten sposób karmić życie duchowe wierzących. On ponadto sprawia, że język łaciński staje się oficjalnym w Kościele, umacniając w ten sposób - według Hieronima - autorytet Katedry Piotra w chrześcijaństwie. Korneliusz jest papieżem, który walczy przeciwko nieprzyjaciołom wewnętrznym i zewnętrznym Kościoła. W jego życiu i działalności uwidacznia się także cnota umiarkowania, równowaga, pierwszeństwo miłosierdzia w powiązaniu z energiczną apologią prawdy i ze zmysłem organizacyjnym wspólnoty kościelnej. Jego męczeństwo przypieczętowuje solidność jego posługi, która umacnia braci w wierze i milości.

Dwaj następni papieże - Damazy i Bonifacy - byli dla Hieronima beatissimi amici. Szczególnie głęboka więź wywiązała się między nim a pierwszym z tych papieży, którego był ścisłym współpracownikiem. Hieronim wysławia zdolności poetyckie i wartości duchowe Damazego. Mnich-egzegeta oddaje się do dyspozycji tegoż papieża-przyjaciela $\mathrm{z}$ całą swą kompetencją wytrawnego biblisty. W stosunku do Bonifacego wspomina dawną ich przyjaźn. W słowach Hieronima dają się tu odczuć tony pożegnania z życiem doczesnym, które już się dopełniło, ale także i radość, iż widzi w biskupie Rzymu osobę otaczaną szacunkiem, która będzie musiała dalej walczyć z błędnowiercami.

Następcami św. Piotra jako defensores fidei przeciwko orygenistom i pelagianom w czasach Hieronima byli szczególnie dwaj papieże - Anastazy (poprzez pośrednictwo Teofila Aleksandryjskiego) i Inocenty, do którego asceta z Betlejem bezpośrednio się odwolał, otrzymując solidarne wsparcie w chwilach największego prześladowania. Niezależnie od tego, czy ci papieże działali energicznie, czy też nie, aby ustrzec prawowierności wiary katolickiej, co powinno być według Hieronima główną cechą pasterza, a szczególnie biskupa rzymskiego, szacunek świętego Doktora względem papieży jako takich nigdy się nie zmniejszy.

Hieronim jawi się więc raz jeszcze jako „vir maxime catholicus" i to nie tylko z tego powodu, iż wytrwał w prawdziwej wierze aż do śmierci, lecz także dlatego, że jego zjednoczenie $z$ następcą św. Piotra zapewniało mu pewność niezachwianego trwania w prawdzie i stanowiło gwarancję jedności. Starał się o to ze wszystkich sił, poświęcając temu cały swój geniusz ludzki i duchowy, wraz $\mathrm{z}$ wyróżniającą go pasją kulturalną $\mathrm{i}$ intelektualną, z poświęceniem godnym prawdziwego chrześcijanina i świętego mnicha. 